\title{
La revue Réanimation change de nom !
}

\section{Our Journal changes its Name!}

\author{
J.-P. Mira · C.-É. Luyt
}

C SRLF et Lavoisier SAS 2016

Chère lectrice, cher lecteur,

Comme vous avez pu le remarquer sur la nouvelle couverture de votre revue, celle-ci-évolue. Le graphisme a changé pour intégrer le nouveau logo et la nouvelle charte graphique de la SRLF. La table des matières, qui était sur la couverture, disparait pour réintégrer l'intérieur de la revue. Ces modifications permettent d'homogénéiser les couvertures des deux revues de la Société. Le second changement est le nouveau nom de votre revue : Réanimation devient Médecine Intensive Réanimation. Ces deux revues de la Société ont des objectifs différents et complémentaires : Annals of Intensive Care, revue anglophone, dont l'impact factor en 2015 a atteint 4,529 et qui a l'ambition d'être une grande revue scientifique destinée à diffuser les nouvelles études de la spécialité, et Médecine Intensive Réanimation, revue francophone qui a l'ambition d'être la revue de référence pour la formation continue des réanimateurs français et aussi de tous nos collègues francophones.

Ce changement de nom obéit à une logique d'évolution de notre spécialité : nous ne pratiquons pas que de la réanimation, mais aussi de la médecine intensive, une fois la phase critique passée et par le biais des unités de soins continus. Notre spécialité ayant pris le nom de « médecine intensive réanimation » (MIR), notre revue, dont le but est l'expression de notre pratique, se trouve ainsi plus en accord avec notre quotidien et notre spécialité d'exercice. De plus, les différentes composantes de notre spécialité ont déjà adopté ce nom : le conseil national professionnel (CNP) de notre spécialité est le CNP-MIR, et le DES qui formera les futurs praticiens de nos services s'appelle DESARMIR (DES commun avec l'anesthésie-réanimation). Enfin, une demande a été faite par le CNU pour changer son libellé en ce sens.

Dans un souci d'harmonisation, il nous a donc semblé logique de modifier le nom de la revue, proposition qui a été adoptée par le comité de rédaction et par le conseil d'administration à l'unanimité.

L'esprit reste bien évidemment le même : une revue de qualité, publiée par Lavoisier, un éditeur investi dans la diffusion du savoir, animée par un rédacteur en chef et un comité de rédaction motivés (qu'ils soient remerciés une fois de plus à cette occasion), et dont le but principal est la publication de mises au point en français, mais aussi d'articles originaux. La revue garde la volonté de publier des articles médicaux, mais aussi de continuer à publier des articles écrits par des paramédicaux, infirmiers ou kinésithérapeutes.

Bonne rentrée à toutes et tous et bonne lecture de ce numéro de Médecine Intensive Réanimation.

Bien amicalement.

J.-P. Mira $(\bowtie)$

CHU Cochin-Saint-Vincent de Paul, site Cochin, 27 rue du

Faubourg Saint-Jacques, F-75014 Paris, France

e-mail : jean-paul.mira@aphp.fr

C.-É. Luyt $(\bowtie)$

Service de réanimation, Institut de cardiologie, groupe hospitalier Pitié-Salpêtrière, 47-83 boulevard de l'Hôpital, F-75013 Paris e-mail : charles-edouard.luyt@aphp.fr 